\title{
Ambulatório remoto de Apoio Matricial de Enfermagem no contexto de pandemia da COVID-19: relato de experiência
}

\author{
Isabela de Souza Santana ${ }^{1}$, Cristiane Chaves de Souza², Laís Sousa da Silva ${ }^{3}$, \\ Bárbara Guimarães Lourenço ${ }^{4}$, Ísis Milani de Sousa Teixeira ${ }^{5}$
}

\begin{abstract}
Resumo: $O$ apoio matricial de enfermagem (AME) é uma estratégia especializada que auxilia as equipes de saúde nas condutas e intervenções de enfermagem. Em sua vertente didático-pedagógica, propõe atualizações em saúde aos profissionais, em busca de promover a educação continuada. Entretanto, com a pandemia da COVID-19, atividades extensionistas foram readaptadas para o modelo remoto. Tem-se por objetivo relatar a experiência da adaptação das atividades presenciais para o regime remoto de um projeto de extensão de ambulatório de apoio matricial, em uma unidade de atenção secundária em saúde. O projeto permitiu a produção e divulgação de quatro Podcasts que visaram abordar diferentes temas relacionados à pandemia, incluindo a temática da vacinação contra a COVID-19. Estes obtiveram um alcance de 146 reproduções, mostrando-se uma estratégia eficaz para a continuidade do intercâmbio entre a extensão universitária e a comunidade em tempos de distanciamento social.
\end{abstract}

Palavras-chave: Enfermagem. Tecnologia. COVID-19.

Área Temática: Saúde.

\section{Remote Outpatient Matrix Support for Nursing in the COVID-19 Pandemic Context: Experience Report}

\begin{abstract}
Matrix support in nursing (AME) is a specialized strategy that helps health teams in nursing behaviors and interventions. In its didactic-pedagogical aspect, it proposes health updates to professionals, seeking to promote continuing education. However, with the COVID-19 pandemic, extension activities were readapted to the remote model. The aim is to report the experience of adapting face-to-face activities to the remote regime of an outpatient extension project for matrix support, in a secondary health care unit. The project allowed the production and dissemination of four Podcasts that aimed to address different issues related to the pandemic, including the issue of vaccination against COVID-19. These had a range of 146 reproductions, proving to be an effective strategy for the continuity of the exchange between the university extension and the community in times of social distance.
\end{abstract}

Keywords: Nursing. Technology. COVID-19.

\section{Soporte de matriz para pacientes ambulatorios remotos para enfermería en el contexto de la pandemia COVID-19: Informe de experiencia}

Resumen: El soporte matricial en enfermería (AME) es una estrategia especializada que ayuda a los equipos de salud en las conductas e intervenciones de enfermería. En su vertiente didáctico-pedagógica, propone actualizaciones de salud a

\footnotetext{
${ }^{1}$ Discente do curso de Enfermagem. Universidade Federal de Viçosa. E-mail: isabela.s.santana @ufv.br

${ }^{2}$ Professora Adjunta. Doutora em Enfermagem. Universidade Federal de Viçosa. Departamento de Medicina e Enfermagem.

${ }^{3}$ Discente do curso de Enfermagem. Universidade Federal de Viçosa.

${ }^{4}$ Discente do curso de Enfermagem. Universidade Federal de Viçosa.

${ }^{5}$ Discente do curso de Enfermagem. Universidade Federal de Viçosa.
} 
los profesionales, buscando promover la educación continua. Sin embargo, con la pandemia de COVID-19, las actividades de extensión se readaptaron al modelo remoto. El objetivo es reportar la experiencia de adaptación de actividades presenciales al régimen remoto de un proyecto de extensión de soporte de matriz ambulatoria en una unidad secundaria de salud. El proyecto permitió la producción y difusión de cuatro Podcasts que tenían como objetivo abordar diferentes temas relacionados con la pandemia, incluido el tema de la vacunación contra COVID-19. Estos tuvieron un abanico de 146 reproducciones, demostrando ser una estrategia eficaz para la continuidad del intercambio entre la extensión universitaria y la comunidad en tiempos de distanciamiento social.

Palabras clave: Enfermería. Tecnología. COVID-19.

\section{INTRODUÇÃO}

O Sistema Único de Saúde (SUS) é dividido em três níveis de atenção à saúde: primária, secundária e terciária. Em especial, a atenção secundária visa atender os pacientes cujos agravos de saúde são de maior complexidade e necessitam de atendimento profissional especializado, o que exige a articulação de vários saberes e profissões. Nesse contexto, a formação dos profissionais de saúde é fundamental para que eles possam desempenhar o trabalho de forma eficiente, articulada e embasada em evidências. Assim, a educação continuada é essencial, pois garante a capacitação e atualização da equipe multiprofissional acerca de novos conhecimentos, procedimentos e tecnologias (JÚNIOR, MOREIRA, 2017).

Dentre as possibilidades de implementação da educação continuada, destaca-se o apoio matricial, com objetivo de auxiliar as equipes de saúde na definição de condutas clínicas de Enfermagem quando precisam de orientação de especialistas. Ou seja, o apoio matricial oferece suporte assistencial e técnico-pedagógico às equipes assistenciais, propiciando espaços de discussão, troca de saberes e reflexão para a prática, o que amplia a qualificação técnica, humanística e social dos profissionais de saúde (CAMPOS, DOMITTI, 2007; JÚNIOR, MOREIRA, 2017).

Dentre os serviços de atenção secundária situados no município de Viçosa, Minas Gerais, destaca-se a Unidade de Atendimento Especializado em Saúde (UAES), pertencente à Universidade Federal de Viçosa (UFV). Na UAES são oferecidos atendimentos $100 \%$ pelo SUS em mais de 28 especialidades médicas, além de Enfermagem, Psicologia e Nutrição.

O Apoio Matricial de Enfermagem (AME) é um projeto de extensão que consiste em um serviço especializado para apoiar as equipes de referência da microrregião de Viçosa, estruturado em duas dimensões. Na dimensão assistencial, são realizadas consultas de Enfermagem a pacientes com necessidade de consulta de especialistas para orientações de cuidado. Na dimensão didático-pedagógica, as atividades visam trabalhar a educação continuada de acordo com as necessidades de capacitação dos profissionais de saúde da rede. O projeto tem uma enorme relevância social para os usuários do SUS e para os profissionais de saúde, pois permite a implementação dos ensinamentos da universidade, colocando à serviço da comunidade todo o conhecimento produzido e à disposição dos profissionais a atualização e qualificação.

Em 2020, entretanto, iniciou-se a pandemia da COVID-19, doença infecciosa causada pelo vírus SARS$\mathrm{CoV}-2$, o que levou à necessidade de adotar o isolamento social. Com isso, as atividades presenciais foram 
interrompidas por tempo indeterminado. Dessa forma, houve a necessidade de adaptar o projeto de extensão ao regime remoto, utilizando uma metodologia apoiada no uso de tecnologias digitais. Esta adaptação objetivou reduzir os impactos do isolamento social na formação acadêmica, e na descontinuidade das atividades de atualização/qualificação dos profissionais atendidos pela dimensão didático-pedagógica do projeto (SOARES et al., 2020).

Dentre as duas dimensões do projeto, optou-se por adaptar as atividades da dimensão didático-pedagógica do projeto para o modo remoto, uma vez que as atividades realizadas nesta dimensão têm cunho educativo, e poderiam ser realizadas com o uso das Tecnologias de Informação e Comunicação (TIC), sem prejuízos ao público receptor do projeto. Soma-se a isto, o fato de que uma das vantagens da utilização das TICs na prática da educação em saúde é que essas são capazes de promover um grande alcance do conhecimento devido a sua rápida disseminação, ultrapassando as barreiras físicas do regime presencial (CAETANO et al., 2021) o que ia ao encontro dos objetivos do trabalho da equipe.

\section{OBJETIVO}

Este manuscrito tem como principal objetivo relatar a experiência de acadêmicos de enfermagem no uso de Tecnologias de Informação e Comunicação para adaptação das atividades de um projeto de extensão ao modo remoto, frente ao cenário imposto pela pandemia da COVID-19.

\section{METODOLOGIA}

Trata-se de um relato de experiência da adaptação de atividades de um projeto de extensão para o modo remoto, cujas atividades foram desenvolvidas entre agosto de 2020 a março de 2021.

Para adaptar as atividades da dimensão didático-pedagógica do AME, optou-se pela estratégia de gravação de Podcasts. Os Podcasts são compreendidos como programas de áudios digitais que podem abranger diversas temáticas. Cada áudio é chamado de episódio e deve possuir uma duração pequena para garantir a adesão dos ouvintes. Sua escolha se fez pertinente por apresentar baixo custo, ausência de impedimentos geográficos e cronológicos, ultrapassar a informação textual, ser uma ferramenta assíncrona e móvel, e ter grande potencial de alcance do público-alvo (BRAGÉ et al., 2020).

Os temas de cada Podcast foram decididos conjuntamente pelos alunos e professora coordenadora do projeto, considerando as necessidades percebidas de capacitação profissional relacionadas à infecção por SARS-CoV-2. Para cada temática, eram elencados nomes de possíveis especialistas com expertise na área do tema central do Podcast. Na sequência, os convites aos mesmos eram realizados por meio de contatos via WhatsApp®. Foram incluídos na lista os contatos dos secretários municipais de saúde e os responsáveis pelo agendamento de consultas na UAES de todos os municípios que compõem a microrregião de Viçosa, além dos grupos de WhatsApp ${ }^{\circledR}$ do Centro Acadêmico de Enfermagem com os docentes do curso, grupos 
representantes de cada uma das turmas de Enfermagem, grupos de docentes do curso de Enfermagem, e lista de contatos pessoais de cada um dos integrantes do projeto. Além disso, o link de acesso ao Podcast também foi divulgado na biografia do Instagram ${ }^{\circledR}$ da coordenadora e da bolsista do projeto. Foram excluídos do próximo envio os contatos de pessoas que manifestaram interesse em não continuar recebendo o conteúdo enviado.

Para gravação dos Podcasts, utilizou-se a plataforma Anchor ${ }^{\circledR}$, de livre acesso e gratuita. Cada gravação possuiu uma durabilidade diferente, com média de 15 minutos. O agendamento da gravação era feito de acordo com a disponibilidade do convidado. Findada a gravação, procedia-se a edição do conteúdo e, previamente à ampla divulgação, o conteúdo passava pela aprovação final do especialista e da coordenadora do projeto. Uma vez aprovado o conteúdo, o link de acesso ao Podcast era divulgado para os estudantes e profissionais de saúde da microrregião de Viçosa, utilizando de diferentes meios de comunicação, como grupos de WhatsApp ${ }^{\circledR}$, Instagram ${ }^{\circledR}$, Spotify ${ }^{\circledR}$ e E-mail.

O primeiro Podcast foi avaliado, e os resultados foram utilizados para melhoria na produção dos episódios seguintes. Para tanto, utilizou-se de um formulário do Google ${ }^{\circledR}$ contendo escalas numéricas de 1 à 5 , onde 1 correspondia a "ruim" e 5 a "excelente", além de local reservado para inserção de comentários e sugestões. Este formulário foi anexado juntamente com o link de acesso ao Podcast no momento de divulgação do mesmo.

\section{RESULTADOS E DISCUSSÃO}

O resultado central do projeto de extensão foi a criação de uma temporada de Podcasts com quatro episódios relacionados à pandemia da COVID-19. O primeiro podcast, "Diabetes Mellitus e COVID-19: relação e cuidados de enfermagem", foi elaborado devido ao alto risco de agravamento do paciente diabético quando infectado pelo novo coronavírus, além de ser uma das doenças crônicas mais tratadas por enfermeiros e profissionais de saúde (TEIXEIRA, FERREIRA, SILVA, 2020). Uma metanálise que englobou 33 estudos apontou que pacientes com Diabetes Mellitus e COVID-19 têm maior risco de mortalidade, com uma razão de chance de 1,90 (IC 95\%: 1,37 e 2,64; p <0,01) quando comparados àqueles infectados pelo SARS-CoV-2, mas que não possuem DM (ANGHEBEM, REGO, PICHETH, 2020).

O segundo episódio, “Biossegurança para profissionais de saúde em tempos de COVID-19”, se deu pela importância das medidas de biossegurança na prevenção da infecção viral, especialmente durante a paramentação e desparamentação dos equipamentos de proteção individual. Nesse sentido, FERIOLI, et al. (2020) destacam que é fundamental que os profissionais tenham conhecimento sobre medidas de precaução para garantir sua proteção durante o cuidado de pacientes com suspeita ou infectados pela COVID-19. Para esta gravação, o grupo convidou uma enfermeira do Serviço de Controle de Infecção Hospitalar, de um hospital de Viçosa, a qual trouxe para o material relatos da prática de enfermagem frente aos desafios e as medidas de segurança adotadas pelos profissionais no contexto de pandemia do novo coronavírus. 
Já o terceiro podcast abordou a temática "Lidando com a ansiedade e o medo de profissionais da saúde infectados por COVID-19: cuidando de quem cuida". Este possui grande relevância devido à angústia e à insegurança gerada pelo atual contexto. Estudos apontam que, dentre as categorias profissionais de saúde que mais se infectaram pelo vírus da COVID-19, estão a de Enfermagem. O Brasil é responsável por um terço do total das mortes destes profissionais devido a infecção por SARS-CoV-2. Até março de 2021, 484.081 profissionais haviam sido infectados, segundo dados do Ministério da Saúde e, além disso, ocorreram cerca de 1,3 mortes por dia, ou seja, uma a cada 19 horas (COFEN, 2021).

Para o podcast, participaram duas alunas e a enfermeira coordenadora do projeto, sendo que a última compartilhou um pouco das experiências vivenciadas como profissional da saúde infectada pelo vírus SARSCoV-2. A professora relatou sobre os aspectos físicos e emocionais envolvidos, enfatizando os medos, ansiedades e angústias trazidos pelo contexto, com o objetivo de incentivar o autocuidado físico e mental em tempos de ameaça à saúde em seu conceito ampliado. Ressalta-se que o contexto pandêmico aumentou consideravelmente os índices de estresse, ansiedade e depressão entre os profissionais da saúde (PRADO et al., 2020).

Por fim, no último podcast, optou-se pela expansão do público-alvo. Este teve como título "Conheça e confie na ciência, vacine-se" e contou com a participação de uma enfermeira e professora do Departamento de Medicina e Enfermagem da UFV, vinculada ao processo de vacinação contra COVID-19. Este Podcast ganhou relevância ímpar, uma vez que foi produzido no início das campanhas de vacinação mundial contra a COVID-19, em um contexto onde pairava a desconfiança da população em relação às vacinas. Este Podcast trouxe informações sobre os tipos de vacinas que estão sendo aplicadas no Brasil até o momento, buscando desmistificar as desinformações disseminadas, além de reforçar a necessidade da manutenção dos cuidados de prevenção e proteção contra o vírus, tendo em vista o desconhecimento científico e a alta propagação de notícias falsas (CASTRO, 2021).

De um modo geral, os Podcasts foram positivos em relação à riqueza de conteúdos atualizados, compartilhados com a comunidade e profissionais de saúde. Além disso, contou-se com a ampliação e valorização da experiência dos participantes do projeto quanto à utilização das TICs como forma de manter o vínculo entre o projeto e a comunidade. Portanto, utilizar a dimensão didático-pedagógica e explorar as plataformas digitais de comunicação para disponibilizar evidências científicas atualizadas contribui com a adoção de práticas de promoção e prevenção em saúde, uma vez que estudo recente aponta a importância de a educação acompanhar a evolução das tecnologias (SOARES et al., 2020).

Cabe ressaltar que o projeto concluiu como importante a oportunidade de experiência que o período pandêmico condicionou ao processo de ensino-aprendizagem no que tange à capacitação e atualização dos profissionais da Rede de Atenção à Saúde. Com isso, estratégias de fácil acesso, flexíveis e didáticas foram requeridas para este processo, tais como: tempo adequado, pensando na sobrecarga de trabalho e "infodemia" vivenciada pelos profissionais; participações especiais com enfermeiras da rede assistencial, fortalecendo o vínculo 
e identificação com o público-alvo; linguagem simples e formato de bate-papo como forma de facilitar o entendimento de qualquer ouvinte e divulgação pelas redes sociais e secretários de saúde da microrregião a fim de atingir o público escolhido.

Nesse processo, notou-se algumas dificuldades em relação ao formato presencial, pois mesmo utilizando de estratégias inovadoras de comunicação, o regime remoto proporciona menor proximidade, ausência de contato visual, dificuldade de firmar vínculo e de usar as contribuições dos ouvintes, o que por vezes, dificulta o compartilhamento bilateral de informações. Dessa forma, nota-se a importância da Educação Permanente em Saúde como uma estratégia para transformar os processos de aprendizagem em saúde.

No processo de avaliação do podcast inaugural, $75 \%$ das respostas do formulário foram avaliadas com nota 5 (excelente) e $25 \%$ com nota 4 (muito bom) tanto o conteúdo quanto a compreensão e aprendizado sobre o tema proposto, sendo a avaliação realizada por $100 \%$ de estudantes de enfermagem. Dentre as sugestões, destacaram-se: "diálogo mais fluido e coloquial" e "transparecer naturalidade". Estes dados possibilitaram ajustar a gravação dos próximos podcasts com as seguintes ações: redução do tempo dos próximos episódios, ampliação do público-alvo e divulgação a partir de trabalhos científicos, a fim de aumentar o alcance dos materiais e a adesão. Isso ampliou as habilidades de reflexão e pensamento crítico dos alunos para adaptarem o projeto ao novo cenário e produzir resultados positivos.

A atualização das informações de cada episódio corroborou com a experiência exitosa dos integrantes do projeto no que se refere ao aprendizado e arcabouço teórico adquirido. Ademais, a gravação dos Podcasts permitiu treinar habilidades de comunicação e diálogo, a fimm de melhorar a dicção e didática, características importantes para a profissão da enfermagem no tangente às capacitações rotineiras realizadas por esses profissionais.

\section{CONCLUSÕES}

Conclui-se a relevância de se adaptar as atividades acadêmicas ao regime remoto durante a pandemia, utilizando-se das tecnologias digitais disponíveis para o desenvolvimento de habilidades técnicas e comportamentais. Com isso, profissionais e estudantes de enfermagem puderam experienciar novas vivências, manter o processo de capacitação profissional realizado pelo serviço de apoio matricial, além de reforçar a importância da articulação entre a universidade e a comunidade no processo de enfrentamento à pandemia, conduzido pelo SUS.

\section{REFERÊNCIAS}

ANGHEBEM, Mauren Isfer; REGO, Fabiane Gomes de Moraes; PICHETH, Geraldo. COVID-19 e Diabetes: a relação entre duas pandemias distintas. $R B A C$, v. 52, n. 2, p. 154-159, 2020.

BISPO JÚNIOR, José Patrício; MOREIRA, Diane Costa. Educação permanente e apoio matricial: formação, vivências e práticas dos profissionais dos núcleos de apoio à saúde da família e das equipes apoiadas.

Cadernos de Saúde Pública, v. 33, n. 9 (e00108116), 2017. 
BRAGÉ, Émilly Giacomelli; RIBEIRO, Lahanna da Silva; RAMOS, Domênica Bossardi; FIALHO, Inara Rahde; ROCHA, Débora Gomes da; BUSATTO, Caroline; LACCHINI, Annie Jeanninne Bisso. Desenvolvimento de um podcast sobre saúde mental na pandemia de COVID-19: um relato de experiência. Brazilian Journal of Health Review, Curitiba, v. 3, n. 4, p. 11368-11376, 2020.

CAETANO, Karina; NISHIDA, Lucas; TAVARES, Raquel; KOSTER, Isabella. Desafios para o trabalho da disseminação científica em saúde pública em contexto de disseminação do coronavírus. Reciis - Revista Eletrônica de Comunicação, Informação \& Inovação em Saúde, Rio de Janeiro, v. 15, n. 1, p. 233248, 2021.

CAMPOS, Gastão Wagner de Sousa; DOMITTI, Ana Carla. Apoio matricial e equipe de referência: uma metodologia para gestão do trabalho interdisciplinar em saúde. Cadernos de Saúde Pública, Rio de Janeiro, v. 23, n. 2, p. 399-407, 2007.

CASTRO, Rosana. Vacinas contra a COVID -19: o fim da pandemia? Physis: Revista de Saúde Coletiva, [S.L.], v. 31. 2021. FapUNIFESP(SciELO).

COFEN. Brasil perde ao menos um profissional de saúde a cada 19 horas para a COVID. COFEN, Brasília, 09 mar. 2021.

FERIOLI, Martina; CISTERNINO, Cecilia; LEO, Valentina; PISANI, Lara; PALANGE, Paolo; NAVA, Stefano. Protecting healthcare workers from SARS-CoV-2 infection: practical indications. Eur Respir Rev, 2020; 29(55): 200068.

PRADO, Amanda Dornelas; PEIXOTO, Bruna Cristina; SILVA, Andréa Mara Bernardes da; SCALIA, Luana Araújo Macedo. A saúde mental dos profissionais de saúde frente à pandemia do COVID-19: uma revisão integrativa. Revista Eletrônica Acervo Saúde, n. 46, p. e4128, 26 jun. 2020.

SOARES, Deisi Cardoso; CECAGNO, Diana; QUADROS, Lenice de Castro Muniz de; SPAGNOLO, Lílian Moura de Lima; CUNHA, Tuany Nunes; FRITZEN, Fabiano Milano. Tecnologias da informação e comunicação na educação em saúde acerca do Coronavírus: relato de experiência. Journal of Nursing and Health, v. 10, n. 4 (e20104027), 2020.

TEIXEIRA, Elizabet; FERREIRA, Darlisom Sousa; SILVA, Lucely Paiva Rodrigues da. Processo de trabalho de profissionais de saúde no contexto do diabetes mellitus: educação permanente online como possibilidade. REH-Revista Educação e Humanidades, volume I, número 1, Jan-Jun, 2020, pág.279-290.

Submetido em: 16/08/2021 Aceito em: 07/10/2021. 